\title{
Conformity checking of LPG Transportation Trucks by Modelling and Simulation
}

\author{
Maryam Gallab ${ }^{1,3, *}$, Hafida Bouloiz $^{2}$, Mohamed Tkiouat $^{1}$ and Emmanuel Garbolino ${ }^{3}$ \\ ${ }^{1}$ LERMA, Engineers Mohammadia School, Mohammed V University, Avenue Ibn Sina BP765, Agdal, Rabat, Morocco. \\ ${ }^{2}$ Industrial Engineering Department, LG2I, National School of Applied Sciences, Ibn Zohr University, - B.P: 1136 Agadir - Morocco. \\ ${ }^{3}$ Crisis and Risk research Centre (CRC), Mines Paris-Tech, 1 rue Claude Daunesse CS 1020706904 Sophia Antipolis Cedex France.
}

\begin{abstract}
LPG Loading / unloading site is considered as a dangerous environment of significant risk, especially during LPG transfer operations. These risks may cause catastrophic dangers such as BLEVE (Boiling Liquid Expanding Vapour Explosion), UVCE (Unconfined Vapour Cloud Explosion), etc. The site consists of a tank (RST) containing LPG and three transfer posts. Two posts allow loading of small carrier tankers 6 tons. The other one transfer post transfer LPG contained in jumbo tankers 20 tons inside the tank. The industrial site at risks chooses to demonstrate the need for a modelling-simulation approach. The aim is to check first compliance and authorization of the truck when it enters the site for loading / unloading, to verify the authorization to make the transfer, and then, to identify the driver and his training to ensure that the transfer operations are going to take place without incident. Sometimes, it may happen that Consignment Operator (CO) is busy or absent and thus the driver operates alone without being authorized, in this case the site risks a loss of control due to a lack of resources. The modelling-simulation will be done using multi-agent systems that will present the drivers, trucks, PO and loading / unloading posts as agents in order to have a model facilitating this checking.
\end{abstract}

\section{Introduction}

LPG (Liquefied Petroleum Gas) is considered as a byproduct of the petrochemical industry, and has become since the 1930s, a powerful energy source [1].

LPG domain has a long history of technological advances and major accidents.

Indeed, LPG sector is considered among the most hazardous industrial sectors based on accidents occurrence statistics. Among these accidents:

- Feyzin in France (1966),

- San Juan Ixuatepec disaster in Mexico (1984),

- Izmit in Turkey (2002).

Latest accidents that touched LPG transportation are centralized on the ARIA database (Research and Information Analysis accidents) such as Bollene accident (2008), Morannes accident (2011), Bastia accident (2014), and Lamentin (2015).

The aim is to check first compliance, conformity and authorization of the truck when it enters the site for loading / unloading, to verify the authorization to make the transfer, and then, to identify the driver and his training to ensure that the transfer operations are going to take place without incident in order to avoid leaks and problems leading to explosions.

This paper is structured as follows: Section 2 presents LPG Loading/Unloading Site. Section 3 shows the hazards and accidents that may generate from nonverification of the trucks and drivers. In section 4 , the proposed model and simulation results are given. Finally, a conclusion and perspectives are provided.

\section{LPG Loading/ Unloading Site}

The field of study is the LPG loading/unloading site. LPG sector in France represents approximately 6,000 industrial sites. These sites are divided into: Deposits bottles, filling centres for filling bottles, and bulk relays which are loaded tankers supplying the customer tanks.

The site assures its supply by jumbo tankers from suppliers (Lavera) and distributes LPG to these customers by small carrier tankers. The site consists of a propane mounded tank, transfer equipment in the pump station area (pump / compressor / piping), a loading / unloading post of jumbo tankers and two loading post of small carrier tankers, and a parking area for tankers and bottles $[2,3]$.

\footnotetext{
Corresponding author: meryam09@gmail.com
} 


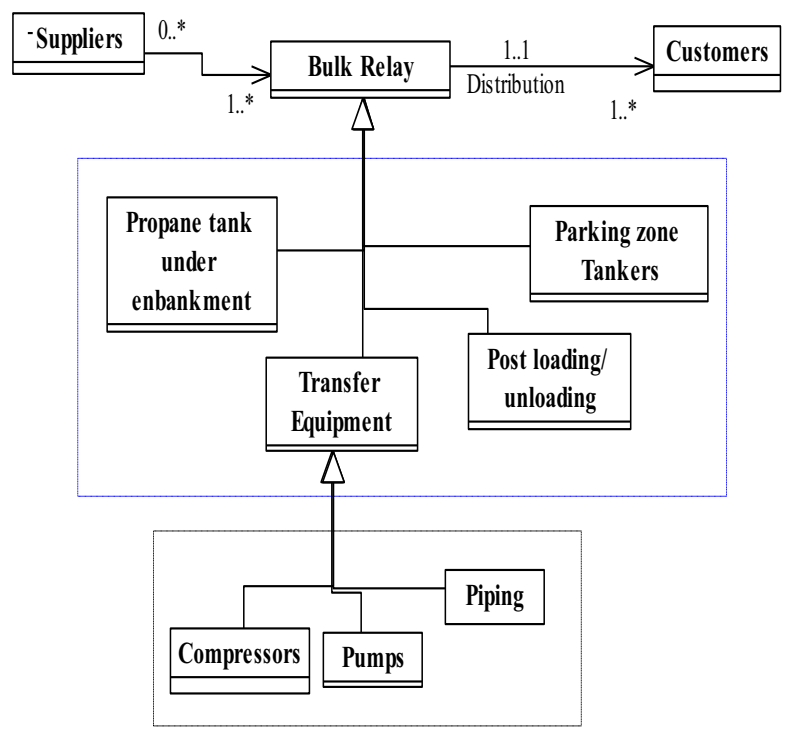

Fig. 1. LPG Loading/Unloading Site.

\subsection{Truck Control Points}

Before the trucks enter the site and perform the loading / unloading operations, a control is carried out at the site entrance by a Consignment Operator $(\mathrm{CO})$ to check the following points:

- Check the driver identification,

- Check permission to load/unload,

- Check the conformity of the tank,

- Check compliance with the quotas,

If all these points are checked and compliant, a $\mathrm{CO}$ provides a bill of loading to the driver, this driver heads towards the transfer station.

\subsection{Checking the driver identification}

The site is in "self-service", drivers perform manipulations to load / unload their trucks. Although everything is automated (depending on the tank, the system knows exactly how many LPG must be injected or aspirated).

A Consignment Operator (CO) monitors operations, especially when unloading jumbo tankers.

The system counts about 400 drivers who can be:

- Trained and audited $\rightarrow$ they can operate in selfservice,

- A driver is simply formed $\rightarrow$ he operates under the $\mathrm{CO}$ supervision,

- The driver is not formed $\rightarrow$ the $\mathrm{CO}$ who will operate and the driver observes.

\section{Hazards generated}

The problem is that failure to verify these points can lead to explosions such as:
- BLEVE (Boiling Liquid Expanding Vapor Explosion): Explosive vaporization of a boiling liquid is a scenario analogous to an explosion induced by the rapid expansion of flammable vapors produced by a gaseous substance preserved under pressure in liquid phase in a medium confined; Of this event, can occur effects of overpressure and thermal irradiations (fireball) for people and structures.


Fig. 2. BLEVE Formation.

- A UVCE (Unconfined Vapour Cloud Explosion) is an accident scenario induced by the reject and the dispersion of flammable substance gas or vapour phase in an unconfined environment, from which may arise, in case of source ignition, thermal and overpressure effects for Humans, structures and the environment. From this explosion, will produce thermal and overpressure effects which depend on local conditions and, specifically, weather and gas mixture conditions.

A UVCE, generally, comprises the following steps: 


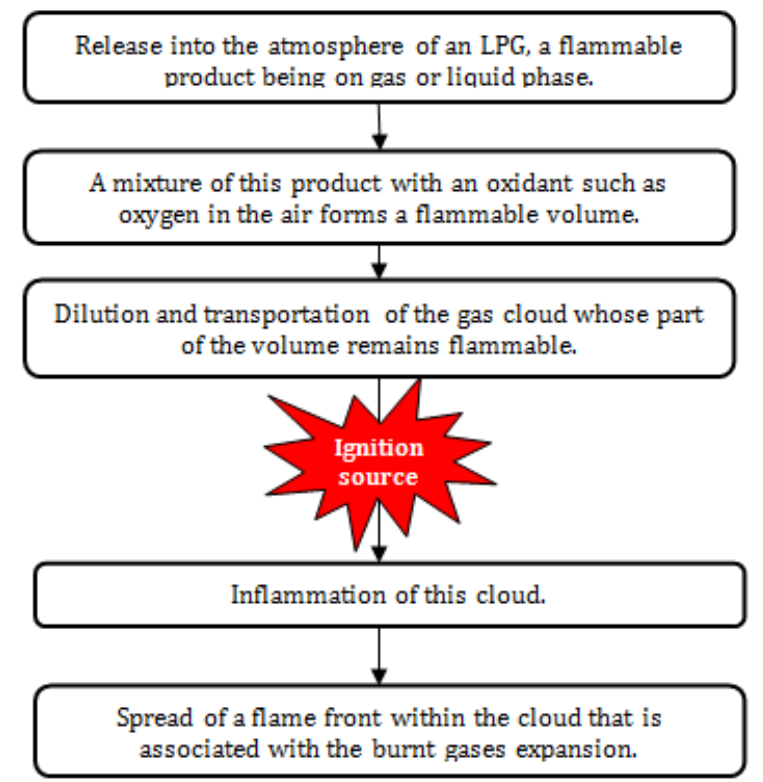

Fig. 3. UVCE Formation.

\section{The proposed Model}

To avoid dangers and to ensure a healthy environment for loading / unloading operations, we propose a model to facilitate verification based on Modelling and Simulation by Multi-Agent Systems.

The simulation by multi-agent systems is adapted to reality, by giving a simple and high quality representation of reality. Multi-Agent Systems are used to find a global solution to a global problem, to model the non-linear, scalable, heterogeneous and complex systems, and to analyse the different interactions between autonomous agents of a global system.

Develop a simulator with AnyLogic platform and preliminary results are presented that will present the drivers, trucks, $\mathrm{CO}$ and loading / unloading posts as agents to have a model facilitating this checking.

\subsection{AnyLogic Platform}

AnyLogic is a simulation platform that includes a graphical modelling language facilitating the models' construction. This platform allows the user performing custom simulation models through its large graphical editor, visual and animated representation (2D and 3D) of the different entities of simulation, and with Java code. Furthermore, it is the first and the only dynamic simulation tool that gathers and combines these threedifferent modelling-simulation approaches: System Dynamics, Discrete event, and Multi-Agent Systems (MAS).

AnyLogic is the most suitable platform for modelling industrial problems especially the one of the supply chain [3-7].

\subsection{Simulation Results}

Simulation model provides checking truck before proceeding to loading/unloading operations in order to ensure compliance of trucks to prevent leaks that could create explosion.

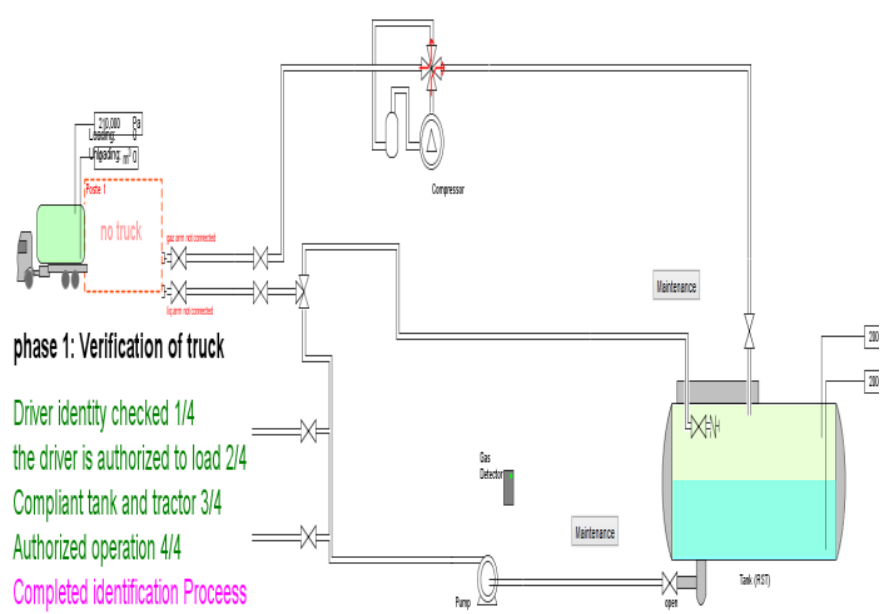

Fig. 4. AnyLogic simulation for checking trucks.

The simulation displays 4 possible cases: Initially when the truck and the tank are complying, we checked the authorization and driver training (Fig. 5-8).

If the driver is trained and audited, it is displayed in green:

\section{Veritication of the truck conformity: T Tractor and tank compliant. \\ Authentication and authorization of the driver". The driver is trained and audilied. He operates alone.}

Fig.5. Checking the tuck conformity and authentication of the driver (a).

If the driver is completely formed, it is displayed in blue:

\section{Verification of the truck conformity: - Tractor and tank compliant. \\ Authentication and authorization of the driver," The driver is completely formed. The $\mathrm{CO}$ monitors.}

Fig.6. Checking the tuck conformity and authentication of the driver (b). 
If the driver is not formed, it is displayed in black (Fig.7):

\section{Verification of the truck conformity: Tractor and tank compliant. \\ Authentication and authorization of the driver." The driver is not formed. The $\mathrm{CO}$ operates.}

Fig.7. Checking the tuck conformity and authentication of the driver (c).

If the truck or tanks are not compliant, it is displayed in red (Fig. 8):

\section{Verification of the truck conformity: improper tractor. d \\ Authentication and authorization of the driver:" The driver is not formed. The $\mathrm{CO}$ operates.}

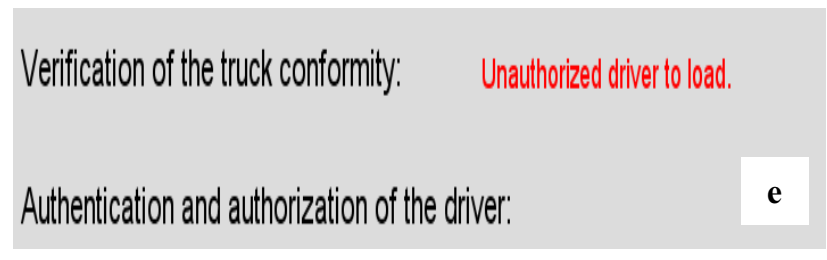

Fig.8. Checking the tuck conformity and authentication of the driver $(\mathrm{d}, \mathrm{e})$.

\section{Conclusion and Perspectives}

LPG Loading/unloading site is considered as a dangerous environment of significant risk, especially during LPG transfer operations. These risks may cause catastrophic dangers such as BLEVE (Boiling Liquid Expanding Vapour Explosion), UVCE (Unconfined Vapour Cloud Explosion), etc.

The modelling-simulation are done using multi-agent systems that present the drivers, trucks, $\mathrm{CO}$ and loading / unloading posts as agents in order to have a model facilitating this checking.

Several other improvements can be made to the proposed model and simulator such as ensuring the safety of maintenance by providing a decision support system, for occupational hazard analysis in maintenance tasks, which will allow orienting the actors to the best decisions in order to minimize hazards that may arise. It is a model for risk management for assessing and simulating risk scenarios related to maintenance activities.

\section{References}

1. S. Lim. Influence lessons learned from accidents involving LPG storage in the development of guides to good practice abroad. Study Report No. DRA-0885166-00650B, 04/09/2008 : INERIS for MEEDDAT / SEI / BARPI (2008).

2. M. Gallab, H. Bouloiz, E. Garbolino, M. Tkiouat, M.A. ElKilani, N. Bureau. Risk analysis of maintenance activities in a LPG supply chain with a Multi-Agent approach. J Loss Prevent Proc, 47, pp 41-56 (2017).

3. M. Gallab, H. Bouloiz, Y. Chater, M. Tkiouat. Towards a simulation Model to ensure the Availability of Machines in Maintenance Activities. Inter. J. of Computer, Electrical, Automation, Control and Information Engineering, 10, $\mathrm{N}^{\circ} 7$ (2016).

4. C. Almeder, M. Preusser. A hybrid Simulation Optimization approach for supply chains. Proceeding EUROSIM/ SLOSIM, Ljubljana, Slovenia. pp. 1-6 (2007).

5. F. Barahona, M. Ettl, M. Petrik, P. Rimshnick. Agile logistics simulation and optimization for managing disaster responses. Proceedings of the Winter Simulation Conference, 978-1-4799-20761/13/\$31.00, IEEE, pp. 3340-3351 (2013).

6. S. Kim, S. Mungle, Y.L. Son. An agent-based simulation approach for dual toll pricing of hazardous material transportation. Proceedings of the Winter Simulation Conference. 978-1-47992076-1/13/\$31.00, IEEE, pp. 2520-2531 (2013).

7. J. Yuan, T. Ponsignon. Towards a SemiConductor Supply Chain Library (SCSC-SIMLIB). Proceedings of the Winter simulation Conference. 978-1-4799-7486-3/14/\$31.00, IEEE, pp. 2522-2532 (2014). 